\title{
Actuator Fault estimation with pole placement constraints for Takagi-Sugeno fuzzy systems with interval time varying delay: An LMI approach
}

\author{
Hamdi Gassara * Ahmed El Hajjaji * Mohamed chaabane ** \\ * Modeling, Information and Systems Laboratory, University of \\ Picardie Jules Verne, MIS, 7 Rue du Moulin Neuf 80000 Amiens \\ France((hamdi.gassara, ahmed.hajjaji)@u-picardie.fr). \\ ** Department of Electrical Engineering, Research Unit of Automatic \\ Control, National School of Engineering of Sfax, University of Sfax, \\ Route Soukra Km 3.5, B.P. 1173, 3038 Sfax, Tunisia
}

\begin{abstract}
This paper adresses the problem of fault estimation for continuous-time TakagiSugeno (T-S) fuzzy systems with interval time varying delay. The approach is based on a fuzzy Adaptive Fault Diagnostic Observer (AFDO). By considering an appropriate Lyapunov function, less conservative linear-matrix-inequality (LMI) conditions for the existence of the fault estimator are proposed. Furthermore, a pole placement is introduced in order to guarantee a faster convergence of estimation errors on the states and the faults. An efficient example is provided to illustrate the effectiveness of the proposed result.
\end{abstract}

Keywords: Linear Matrix Inequalities, Takagi-Sugeno fuzzy systems, Fault estimation, Adaptive fuzzy observer

\section{INTRODUCTION}

As is well known, Takagi-Sugeno(TS) Fuzzy observer design is become an active research field owing to its particular importance in observer-based control, fault diagnosis, and Fault Tolerant Control(FTC)of the nonlinear systems (Zhang and al. (2002), Chadli and El Hajjaji (2006), Kim and Lee (2000), Boukas and El Hajjaji (2006), Oudghiri M. and al (2008)). Different design techniques of TS fuzzy observer are been developed in the literature (Akhenak (2004), D. Ichalal and al. (2010), D. Ichalal and al. (2009), Chadli M. and al (2009), Bouattour M. and al (2010)). Among these techniques, we can find the Adaptive Fault Diagnostic Observer (AFDO) technique which allow to estimate the state vector and actuator fault simultaneously (Jiang and al. (2006), Zhang and al. (2008), Zhang and al. (2009)).

On the other hand, time delay is one of the instability sources in dynamical processus. For this reason, T-S fuzzy model has been extended to deal with nonlinear systems with time delay. Different delay-independent methodologies have been proposed for analysis and synthesis for TS fuzzy systems with time delay (Chen and Liu (2005), Lee and al. (2000), Xu and Lam (2005)). It is generally recognized that delay-dependent results are usually less conservative than delay-independent ones. That's why, delay-dependent techniques have been reported in ( $\mathrm{Li}$ and al. (2004), Guan and Chen (2004), Chen and Liu (2005)). Considering the AFDO design, the problem of fault estimation for linear system are treated in Zhang and al. (2008). This idea is extended to deal with T-S fuzzy models with constant time delay in Zhang and al. (2009). The obtained result is delay-independent. By adopting the free weighting matrix technique, a delay-dependent results are obtained for linear system with bounded time delay Jiang and al. (2009). However, the use of too many free weighting matrices makes the design method more complicate . To the best of our knowledge, so far, the problem of fault estimation of T-S fuzzy systems with interval time varying delay has not been addressed in the literature.

Motivated by the aforementioned observation, in this paper, we study the fault estimation for Takagi-Sugeno fuzzy systems with interval time varying delay based on AFDO. A pole placement is introduced in order to deliver sufficiently fast and well-responses of fault estimation.

Notations $W+W^{T}$ is denoted as $W+(*)$ for simplicity.

\section{PROBLEM FORMULATION AND PRELIMINARIES}

Consider an T-S fuzzy system with time-varying delay. The $i^{t h}$ rule of the system is expressed in the following IF-THEN rules.

Plant Rule $i(i=1,2, \cdots, r)$ : If $\theta_{1}$ is $\mu_{i 1}$ and $\cdots$ and $\theta_{p}$ is $\mu_{i p}$ THEN

$$
\begin{aligned}
\dot{x}(t)= & A_{i} x(t)+A_{\tau i} x(t-\tau(t))+B_{i} u(t) \\
& +E_{i} f(t) \\
y(t)= & C x(t)
\end{aligned}
$$

where $\theta_{j}(x(t))$ and $\mu_{i j}(i=1, \cdots, r, j=1, \cdots, p)$ are respectively the premise variable and the fuzzy sets; $\psi(t)$ is the initial conditions; $x(t) \in \Re^{n_{x}}$ is the state; $u(t) \in \Re^{n_{u}}$ 
is the control input; $f(t) \in \Re^{n_{f}}$ represents the actuator fault vector. It is assumed that the derivative of $f(t)$ with respect to time is norm bounded, i.e. $\|\dot{f}(t)\| \leq f_{1}$ and $0 \leq f_{1}<\infty . r$ is the number of IF-THEN rules.

the interval time varying delay satisfies

$$
0<\tau_{m} \leq \tau(t) \leq \tau_{M}, \dot{\tau}(t) \leq \beta
$$

Denote

$$
\tau_{1}=\frac{\tau_{m}+\tau_{M}}{2} ; \tau_{2}=\frac{\tau_{M}-\tau_{m}}{2}
$$

The AFDO is constructed as

Observer Rule $i(i=1,2, \cdots, r)$ : If $\theta_{1}$ is $\mu_{i 1}$ and $\cdots$ and $\theta_{p}$ is $\mu_{i p}$ THEN

$$
\begin{aligned}
\dot{\hat{x}}(t)= & A_{i} \hat{x}(t)+A_{\tau i} \hat{x}(t-\tau(t))+B_{i} u(t) \\
& +E_{i} \hat{f}(t)-L_{i}(\hat{y}(t)-y(t)) \\
\hat{y}(t)= & C \hat{x}(t)
\end{aligned}
$$

Denote $e_{x}(t)=\hat{x}(t)-x(t), e_{y}(t)=\hat{y}(t)-y(t), e_{f}(t)=$ $\hat{f}(t)-f(t)$

then the error dynamic is given by

$$
\begin{aligned}
\dot{e}_{x}(t)= & {\left[(A(t)-L(t) C) e_{x}(t)\right.} \\
& \left.+A_{\tau}(t) e_{x}(t-\tau(t))+E(t) e_{f}(t)\right] \\
e_{y}(t)= & C e_{x}(t)
\end{aligned}
$$

where

$$
A(t)=\sum_{i=1}^{r} h_{i} A_{i} ; A_{\tau}(t)=\sum_{i=1}^{r} h_{i} A_{\tau i} ; E(t)=\sum_{i=1}^{r} h_{i} E_{i}
$$

\section{RESULT}

In this section, we first present useful lemma and then present the main result.

Lemma 1. For a symmetric positive definite matrix $P$, the following inequality holds

$$
2 x^{T} y \leq x^{T} P x+y^{T} P^{-1} y
$$

Lemma 2. Consider a negative definite matrix $\Pi<0$.

Given a symmetric matrix $X$ of appropriate dimension such that $X^{T} \Pi X<0$, then, $\exists \lambda \in \Re^{+}$such that

$$
X^{T} \Pi X \leq-2 \lambda X-\lambda^{2} \Pi^{-1}
$$

Lemma 3. Given matrices $M, E, F(t)$ with compatible dimensions and $F(t)$ satisfying $F(t)^{T} F(t) \leq I$.

Then, the following inequalities hold for any $\epsilon>0$

$M F(t) E+E^{T} F(t)^{T} M^{T} \leq \epsilon M M^{T}+\epsilon^{-1} E^{T} E$

Definition 1. A subset $D$ of the complex plane is called an LMI region if there exist a symmetric matrix $\alpha$ and a matrix $\beta$ such that

$$
D=\left\{z \in \mathcal{C}, f_{D}(z)<0\right\}
$$

with

$$
f_{D}(z)=\alpha+\beta z+\beta^{T} \bar{z}
$$

A dynamical system is called $D$ stable if all its poles lie in $D$ (that is, all eigenvalues of the matrix $A$ lie in $\mathrm{D}$ ).

Lemma 4. Chilali and Gahinet (1996) $A$ is $D$ stable if and only if there exists a symmetric matrix $P>0$ such that

$$
\alpha \otimes P+\beta \otimes A P+\beta^{T} \otimes P A^{T}<0
$$

where $\otimes$ denotes the Kronecker product of matrices

Theorem 1. For given positif scalar $\lambda$, if there exist symmetric positive definite matrices $P, Q_{l}(l=1,2,3,4,5)$, $R_{m}(m=1,2,3,4), M$ and matrices $Y i$ and $F_{i}$ such that the following LMI hold

$$
\begin{gathered}
\alpha \otimes P+\beta \otimes A_{i}^{T} P-\beta \otimes C^{T} Y_{i}^{T} \\
+\beta^{T} \otimes P A_{i}-\beta^{T} \otimes Y_{i} C<0, i=1,2, \cdots, r \\
\Xi_{i j}+\Xi_{j i}<0, i, j=1,2, \cdots, r, i \leq j \\
E_{i}^{T} P=F_{i} C, i=1,2, \cdots, r
\end{gathered}
$$

then $A(t)-L(t) C$ is $D$ stable and the fuzzy Fast Adaptive Fault Estimation (FAFE) algorithm

$$
\dot{\hat{f}}(t)=-\Gamma \sum_{i=1}^{r} h_{i} F_{i}\left(\dot{e}_{y}(t)+e_{y}(t)\right)
$$

can realize that $e_{x}(t)$ and $e_{f}(t)$ are uniformly ultimately bounded.

In this case, the $L_{i}$ are given by

$$
\begin{gathered}
L_{i}=P^{-1} Y_{i} \\
\Xi_{i j}=\left[\begin{array}{cccc}
\xi_{i j}^{11} & R_{4} & P A_{\tau i} & 0 \\
* & \xi_{22} & R_{3} & Q_{2}+R_{1} \\
* & * & \xi_{33} & 0 \\
* & * & * & \xi_{44} \\
* & * & * & * \\
* & * & * & * \\
* & * & * & * \\
0 & & \xi_{i j}^{16} & \xi_{i j}^{17} \\
0 & & 0 & 0 \\
R_{3} & -A_{\tau i}^{T} P E_{j} & A_{\tau i}^{T} P \\
Q_{2}+R_{2} & 0 & 0 \\
\xi_{55} & & 0 & 0 \\
* & & \xi_{i j}^{66} & E_{i}^{T} P \\
* & & * & \xi_{i j}^{77}
\end{array}\right]
\end{gathered}
$$

where

$$
\begin{aligned}
& \xi_{i j}^{11}=P A_{i}-Y_{i} C+A_{i}^{T} P-C^{T} Y_{i}^{T}+Q_{4}+Q_{5}-R_{4} ; \\
& \xi_{i j}^{16}=-A_{i}^{T} P E_{j}+C^{T} Y_{i}^{T} E_{j} ; \\
& \xi_{i j}^{17}=A_{i}^{T} P-C^{T} Y_{i}^{T} \\
& \xi^{22}=Q_{1}-Q_{5}-R_{1}-R_{3}-R_{4} ; \\
& \xi^{33}=-(1-\beta) Q_{4}-2 R_{3} ; \\
& \xi^{44}=Q_{3}-Q_{1}-R_{1}-R_{2} ; \\
& \xi^{55}=-Q_{3}-R_{2}-R_{3} ;
\end{aligned}
$$




$$
\begin{aligned}
\xi_{i j}^{66}= & -E_{i}^{T} P E_{j}-E_{j}^{T} P E_{i}+M \\
\xi_{i j}^{77}= & -2 \lambda P \\
& +\lambda^{2}\left(\left(\tau_{2}\right)^{2} R_{1}+\left(\tau_{2}\right)^{2} R_{2}+\left(2 \tau_{2}\right)^{2} R_{3}+\left(\tau_{m}\right)^{2} R_{4}\right)
\end{aligned}
$$

\section{Proof:}

Constraints (4): By using lemma (4), $A(t)-L(t) C$ is $D$ stable if and only if there exist a symmetric matrix $P$ such that

$$
\begin{aligned}
& \alpha \otimes P+\beta \otimes(A(t)-L(t) C) P \\
& +\beta^{T} \otimes P(A(t)-L(t) C)^{T}<0
\end{aligned}
$$

which can be rewritten as

$$
\begin{array}{r}
\sum_{i=1}^{r} h_{i}\left[\alpha \otimes P+\beta \otimes\left(A_{i}-L_{i} C\right) P\right. \\
\left.+\beta^{T} \otimes P\left(A_{i}-L_{i} C\right)^{T}\right]<0
\end{array}
$$

Since the solution of $\operatorname{det}\left(A_{i}-L_{i} C\right)=0$ is the same as that $\operatorname{det}\left(\left(A_{i}-L_{i} C\right)^{T}\right)=0$, as long as $D$ stability is the only concern, (10) is equivalent to

$$
\begin{array}{r}
\sum_{i=1}^{r} h_{i}\left[\alpha \otimes P+\beta \otimes\left(A_{i}-L_{i} C\right)^{T} P\right. \\
\left.+\beta^{T} \otimes P\left(A_{i}-L_{i} C\right)\right]<0
\end{array}
$$

Therefore, if (4) hold, then $(A(t)-L(t) C)$ is $D$ stable.

For constraints (5) and (6), Consider the following Lyapunov function

$$
V(t)=\sum_{i=1}^{6} V_{i}(t)
$$

where

$$
\begin{aligned}
V_{1}(t)= & e_{x}(t)^{T} P e_{x}(t)+\int_{t-\tau_{1}}^{t-\tau_{m}}\left\{e_{x}(s)^{T} Q_{1} e_{x}(s)\right. \\
& +2 e_{x}(s)^{T} Q_{2} e_{x}\left(s-\tau_{2}\right) \\
& \left.+e_{x}\left(s-\tau_{2}\right)^{T} Q_{3} e_{x}\left(s-\tau_{2}\right)\right\} d s \\
& +\int_{t-\tau(t)}^{t} e_{x}(s)^{T} Q_{4} e_{x}(s) d s \\
& +\int_{t-\tau_{m}}^{t} e_{x}(s)^{T} Q_{5} e_{x}(s) d s \\
V_{2}(t) & =\tau_{2} \int_{-\tau_{1}}^{-\tau_{m}} \int_{t+\sigma}^{t} \dot{e}_{x}(s)^{T} R_{1} \dot{e}_{x}(s) d s d \sigma \\
V_{3}(t) & =\tau_{2} \int_{-\tau_{M}}^{t} \int_{t+\sigma}^{t} \dot{e}_{x}(s)^{T} R_{2} \dot{e}_{x}(s) d s d \sigma
\end{aligned}
$$

$$
\begin{aligned}
& V_{4}(t)=2 \tau_{2} \int_{-\tau_{M}}^{-\tau_{m}} \int_{t+\sigma}^{t} \dot{e}_{x}(s)^{T} R_{3} \dot{e}_{x}(s) d s d \sigma \\
& V_{5}(t)=\tau_{m} \int_{-\tau_{m}}^{0} \int_{t+\sigma}^{t} \dot{e}_{x}(s)^{T} R_{4} \dot{e}_{x}(s) d s d \sigma \\
& V_{6}(t)=e_{f}(t)^{T} \Gamma^{-1} e_{f}(t)
\end{aligned}
$$

Taking the derivation of $V(t)$, we have

$$
\dot{V}_{1}(t)=2 e_{x}(t)^{T} P \dot{e}_{x}(t)+\Delta
$$

where

$$
\begin{gathered}
\Delta=e_{x}\left(t-\tau_{m}\right)^{T} Q_{1} e_{x}\left(t-\tau_{m}\right)+2 e_{x}\left(t-\tau_{m}\right) Q_{2} e_{x}\left(t-\tau_{1}\right) \\
+e_{x}\left(t-\tau_{1}\right) Q_{3} e_{x}\left(t-\tau_{1}\right)-e_{x}\left(t-\tau_{1}\right)^{T} Q_{1} e_{x}\left(t-\tau_{1}\right) \\
-2 e_{x}\left(t-\tau_{1}\right)^{T} Q_{2} e_{x}\left(t-\tau_{M}\right)-e_{x}\left(t-\tau_{M}\right)^{T} Q_{3} x\left(t-\tau_{M}\right) \\
+e_{x}(t)^{T} Q_{4} e_{x}(t)-(1-\dot{\tau}(t)) e_{x}(t-\tau(t)) Q_{4} e_{x}(t-\tau(t)) \\
+e_{x}(t)^{T} Q_{5} e_{x}(t)-e_{x}\left(t-\tau_{m}\right)^{T} Q_{5} e_{x}\left(t-\tau_{m}\right) \\
\dot{V}_{2}(t)=\tau_{2}\left\{\tau_{2} \dot{e}_{x}(t)^{T} R_{1} \dot{e}_{x}(t)\right. \\
\left.\left.-\int_{t-\tau_{1}}^{t-\tau_{m}} \dot{e}_{x}(s)^{T} R_{1} \dot{e}_{(} s\right) d s\right\}
\end{gathered}
$$

Similarly, we obtain

$$
\begin{aligned}
\dot{V}_{3}(t)= & \tau_{2}\left\{\tau_{2} \dot{e}_{x}(t)^{T} R_{2} \dot{e}_{x}(t)\right. \\
& \left.-\int_{t-\tau_{M}}^{t-\tau_{1}} \dot{e}_{x}(s)^{T} R_{2} \dot{e}_{x}(s) d s\right\} \\
\dot{V}_{4}(t)= & 2 \tau_{2}\left\{2 \tau_{2} \dot{e}_{x}(t)^{T} R_{3} \dot{e}_{x}(t)\right. \\
& \left.-\int_{t-\tau_{M}}^{t-\tau_{m}} \dot{e}_{x}(s)^{T} R_{3} \dot{e}_{x}(s) d s\right\} \\
\dot{V}_{5}(t)= & \tau_{m}\left\{\tau_{m} \dot{e}_{x}(t)^{T} R_{4} \dot{e}_{x}(t)\right. \\
& \left.-\int_{t-\tau_{m}}^{t} \dot{e}_{x}(s)^{T} R_{4} \dot{e}_{x}(s) d s\right\} \\
\dot{V}_{6}(t)= & -2 e_{f}(t)^{T} F(t) C\left(\dot{e}_{x}(t)+e_{x}(t)\right) \\
& -2 e_{f}(t)^{T} \Gamma^{-1} \dot{f}(t)
\end{aligned}
$$

By considering equality (6), we obtain:

$$
\begin{aligned}
& \dot{V}_{1}(t)+\dot{V}_{6}(t)= \\
& 2 e_{x}(t)^{T} P\left[(A(t)-L(t) C) e_{x}(t)+A_{\tau}(t) e_{x}(t-\tau(t))\right] \\
& +\Delta-2 e_{f}(t)^{T} F(t) C \dot{e}_{x}(t)-2 e_{f}(t)^{T} \Gamma^{-1} \dot{f}(t)
\end{aligned}
$$

From lemma (1), we can obtain that

$$
\begin{aligned}
& -2 e_{f}(t)^{T} \Gamma^{-1} \dot{f}(t) \leq \\
& e_{f}(t)^{T} M e_{f}(t)+\dot{f}(t)^{T} \Gamma^{-1} M^{-1} \Gamma^{-1} \dot{f}(t) \leq \\
& e_{f}(t)^{T} M e_{f}(t)+\delta
\end{aligned}
$$


where

$$
\delta=f_{1}^{2} \lambda_{\max }\left(\Gamma^{-1} M^{-1} \Gamma^{-1}\right)
$$

Applying jessen's inequality Gu and al. (2003) to deal with the cross product items, we have

$$
\begin{aligned}
& -\tau_{2} \int_{t-\tau_{1}}^{t-\tau_{m}} \dot{e}_{x}(s)^{T} R_{1} \dot{e}_{x}(s) d s \\
& \leq\left[\begin{array}{c}
e_{x}\left(t-\tau_{m}\right) \\
e_{x}\left(t-\tau_{1}\right)
\end{array}\right]^{T}\left[\begin{array}{cc}
-R_{1} & R_{1} \\
* & -R_{1}
\end{array}\right]\left[\begin{array}{c}
e_{x}\left(t-\tau_{m}\right) \\
e_{x}\left(t-\tau_{1}\right)
\end{array}\right] \\
& -\tau_{2} \int_{t-\tau_{M}}^{t-\tau_{1}} \dot{e}_{x}(s)^{T} R_{2} \dot{e}_{x}(s) d s \\
& \leq\left[\begin{array}{c}
e_{x}\left(t-\tau_{1}\right) \\
e_{x}\left(t-\tau_{M}\right)
\end{array}\right]^{T}\left[\begin{array}{cc}
-R_{2} & R_{2} \\
* & -R_{2}
\end{array}\right]\left[\begin{array}{c}
e_{x}\left(t-\tau_{1}\right) \\
e_{x}\left(t-\tau_{M}\right)
\end{array}\right]
\end{aligned}
$$$$
-2 \tau_{2} \int_{t-\tau_{M}}^{t-\tau_{m}} \dot{e}_{x}(s)^{T} R_{3} \dot{e}_{x}(s) d s
$$$$
\leq-\left(\tau(t)-\tau_{m}\right) \int_{t-\tau(t)}^{t-\tau_{m}} \dot{e}_{x}(s)^{T} R_{3} \dot{e}_{x}(s) d s
$$$$
-\left(\tau_{M}-\tau(t)\right) \int_{t-\tau_{M}}^{t-\tau(t)} \dot{e}_{x}(s)^{T} R_{3} \dot{e}_{x}(s) d s
$$$$
\leq\left[\begin{array}{c}
e_{x}\left(t-\tau_{m}\right) \\
e_{x}(t-\tau(t)) \\
e_{x}\left(t-\tau_{M}\right)
\end{array}\right]^{T}\left[\begin{array}{ccc}
-R_{3} & R_{3} & 0 \\
* & -2 R_{3} & R_{3} \\
* & * & -R_{3}
\end{array}\right]
$$$$
\left[\begin{array}{c}
e_{x}\left(t-\tau_{m}\right) \\
e_{x}(t-\tau(t)) \\
e_{x}\left(t-\tau_{M}\right)
\end{array}\right]
$$

$$
\begin{aligned}
& -\tau_{m} \int_{t-\tau_{m}}^{t} \dot{e}_{x}(s)^{T} R_{4} \dot{e}_{x}(s) d s \\
& \leq\left[\begin{array}{c}
e_{x}(t) \\
e_{x}\left(t-\tau_{m}\right)
\end{array}\right]^{T}\left[\begin{array}{cc}
-R_{4} & R_{4} \\
* & -R_{4}
\end{array}\right]\left[\begin{array}{c}
e_{x}(t) \\
e_{x}\left(t-\tau_{m}\right)
\end{array}\right]
\end{aligned}
$$

Let $\eta(t)=\left[e_{x}(t)^{T}, e_{x}\left(t-\tau_{m}\right)^{T}, e_{x}(t-\tau(t))^{T}, e_{x}(t-\right.$ $\left.\left.\tau_{1}\right)^{T}, e_{x}\left(t-\tau_{M}\right)^{T}, e_{f}(t)^{T}\right]^{T}$.

$$
\dot{V}(t) \leq \eta(t)^{T} \Omega(t) \eta(t)
$$

$$
+\dot{e}_{x}(t)^{T}\left(\left(\tau_{2}\right)^{2} R_{1}+\left(\tau_{2}\right)^{2} R_{2}+\left(2 \tau_{2}\right)^{2} R_{3}+\left(\tau_{m}\right)^{2} R_{4}\right) \dot{e}_{x}(t)+\delta
$$

where

$$
\Omega(t)=\left[\begin{array}{ccc}
\omega_{11}(t) & R_{4} & P A_{\tau}(t) \\
* & \xi_{22} & R_{3} \\
* & * & \xi_{33} \\
* & * & * \\
* & * & * \\
* & * & *
\end{array}\right.
$$

$$
\left.\begin{array}{ccc}
0 & 0 & \omega_{16}(t) \\
Q_{2}+R_{1} & 0 & 0 \\
0 & R_{3} & A_{\tau}(t)^{T} P E(t) \\
\xi_{44} & -Q_{2}+R_{2} & 0 \\
* & \xi_{55} & 0 \\
* & * & \omega_{66}(t)
\end{array}\right]
$$

in which

$$
\begin{aligned}
\omega_{11}(t)= & P(A(t)-L(t) C)+(A(t)-L(t) C)^{T} P \\
& +Q_{4}+Q_{5}-R_{4} ; \\
\omega_{16}(t)= & -(A(t)-L(t) C)^{T} P E(t) ; \\
\omega_{66}(t)= & -2 E(t)^{T} P E(t)+M
\end{aligned}
$$

By the Schur complement, we obtain

$$
\Phi(t)=\left[\begin{array}{cc}
\Omega(t) & \phi_{12}(t) \\
* & -P\left(\left(\tau_{2}\right)^{2} R_{1}+\left(\tau_{2}\right)^{2} R_{2}\right. \\
& \left.+\left(2 \tau_{2}\right)^{2} R_{3}+\left(\tau_{m}\right)^{2} R_{4}\right)^{-1} P
\end{array}\right]
$$

where

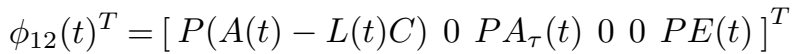

Applying lemma (2), if $\Xi_{i j}+\Xi_{j i}<0, i, j=1,2, \cdots, r, i \leq$ $j$, then there exist a scalar $\epsilon>0$ such that $\dot{V}(t)<$ $-\epsilon\|\eta(t)\|^{2}+\delta$. It follows that $\dot{V}(t)<0$ for $\epsilon\|\eta(t)\|^{2}>\delta$, which means that $\eta(t)$ converges to a small set $S=$ $\left\{\eta(t) \mid\|\eta(t)\|^{2} \leq \frac{\delta}{\epsilon}\right\}$ according to Lyapunov stability theory. Therefore, estimation errors of both the state and the fault are uniformly ultimately bounded.

Remark 1. The purpose of introducing the pole placement constraints is to improve the transient performance of fault estimation.

Remark 2. Zhang and al. (2008) It is easy to solve the inequalities (4)-(5) by using LMI Toolbox. For equation (6), we can make a transform into the following optimization problem

Minimize $\rho>0$

Subject to

$$
\left[\begin{array}{cc}
\rho I & E_{i}^{T} P-F_{i} C \\
* & \rho I
\end{array}\right]>0, i=1, \cdots, r
$$

Remark 3. Examples of LMI region :

- Half-plane $\operatorname{Re}(s)<-a$. In this case, (4) can be rewritten as

$$
2 a P+A_{i}^{T} X-C^{T} Y_{i}^{T}+X A_{i}-Y_{i} C<0
$$

- Disk centred at $(-q, 0)$ with radius $r$. In this case, (4) can be rewritten as

$$
\left[\begin{array}{c}
-r P-r P+A_{i}^{T} P-C^{T} Y_{i}^{T} \\
-r P
\end{array}\right]<0
$$

- Conic sector with apex at the origin and inner angle $2 \theta$. In this case, (4) can be rewritten as 


$$
\begin{gathered}
{\left[\begin{array}{c}
\sin (\theta)\left(A_{i}^{T} P-C^{T} Y_{i}^{T}+P A_{i}-Y_{i} C\right) \\
-\cos (\theta)\left(A_{i}^{T} P-C^{T} Y_{i}^{T}-P A_{i}+Y_{i} C\right) \\
\cos (\theta)\left(A_{i}^{T} P-C^{T} Y_{i}^{T}-P A_{i}+Y_{i} C\right) \\
\sin (\theta)\left(A_{i}^{T} P-C^{T} Y_{i}^{T}+P A_{i}-Y_{i} C\right)
\end{array}\right]<0}
\end{gathered}
$$

\section{SIMULATION RESULT}

Consider the following T-S fuzzy model

$$
\dot{x}(t)=\sum_{i=1}^{2} h_{i}\left[A_{i} x(t)+A_{\tau i} x(t-\tau(t))+B_{i} u(t)+E_{i} f(t)\right]
$$

The membership functions for rules 1 and 2 are:

$$
h_{1}\left(x_{2}(t)\right)=1-\frac{x_{2}(t)^{2}}{2.25}, h_{2}\left(x_{2}(t)\right)=1-h_{1}\left(x_{2}(t)\right)(22)
$$

where

$$
\begin{gathered}
A_{1}=\left[\begin{array}{cc}
-0.1125 & -0.0200 \\
1 & 0
\end{array}\right], A_{2}=\left[\begin{array}{cc}
-0.1125 & -1.5270 \\
1 &
\end{array}\right] \\
A_{\tau 1}=\left[\begin{array}{cc}
-0.0125 & -0.0050 \\
0 & 0
\end{array}\right], A_{\tau 2}=\left[\begin{array}{cc}
-0.0125 & -0.2300 \\
0 & 0
\end{array}\right] \\
E_{1}=E_{2}=B_{1}=B_{2}=\left[\begin{array}{l}
1 \\
1
\end{array}\right], C=\left[\begin{array}{ll}
0 & 1
\end{array}\right]
\end{gathered}
$$

By solving the conditions in theorem 1, without pole placement, we obtain

$$
\begin{gathered}
F=10^{3} \times 1.8555 \\
L_{1}=\left[\begin{array}{l}
1.7056 \\
2.1580
\end{array}\right], L_{2}=\left[\begin{array}{l}
0.1081 \\
2.1555
\end{array}\right]
\end{gathered}
$$

First, it assumed that a constant fault $f_{1}(t)$ is created as

$$
\begin{aligned}
f_{1}(t) & =0, \quad 0 \leq t<5 \\
& =20, \quad 5 \leq t \leq 20
\end{aligned}
$$

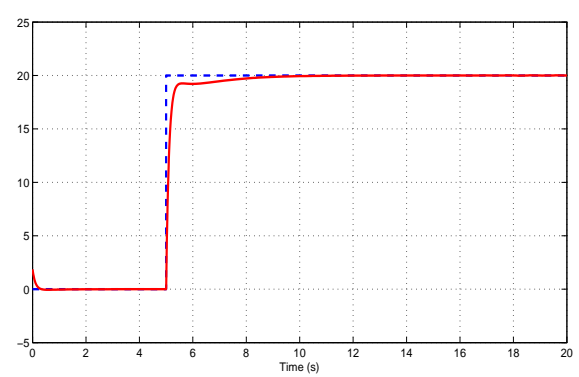

Fig. 1. Fault $f_{1}(t)$ (Dotted) and its estimated $\hat{f}_{1}(t)$ (solid) without pole placement

Then, a time-varying fault is simulated

$$
\begin{array}{rlrl}
f_{2}(t) & =0, & & 0 \leq t<5 \\
& =10 \sin (t-5), & 5 \leq t \leq 20
\end{array}
$$

By solving the conditions in theorem 1 , with pole clustering in the region $\operatorname{Re}(s)<-1$, we obtain

$$
\begin{gathered}
F=10^{3} \times 4.0686 \\
L_{1}=\left[\begin{array}{l}
2.2594 \\
2.3831
\end{array}\right], L_{2}=\left[\begin{array}{l}
0.6692 \\
2.3631
\end{array}\right]
\end{gathered}
$$

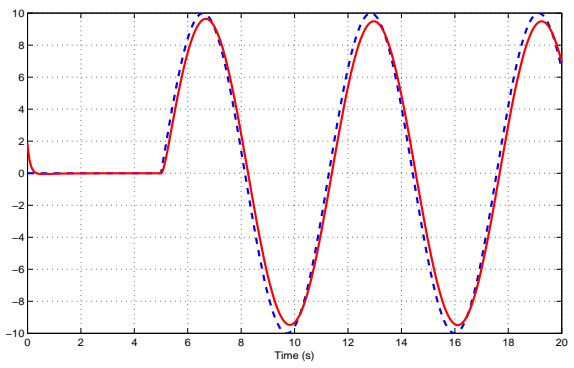

Fig. 2. Fault $f_{2}(t)$ (Dotted) and its estimated $\hat{f}_{2}(t)$ (solid) without pole placement

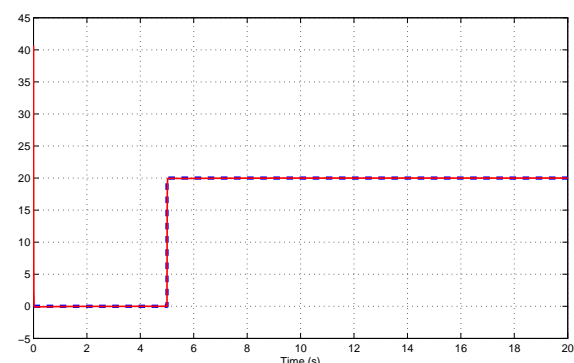

Fig. 3. Fault $f_{1}(t)$ (Dotted) and its estimated $\hat{f}_{1}(t)$ (solid) with pole placement

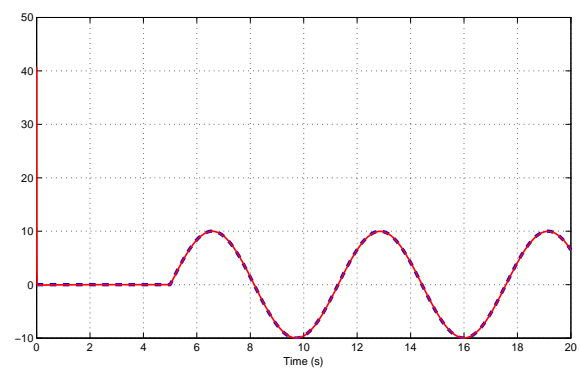

Fig. 4. Fault $f_{2}(t)$ (Dotted) and its estimated $\hat{f}_{2}(t)$ (solid) with pole placement

\section{CONCLUSION}

The problem of delay dependent AFDO design to a class of T-S fuzzy systems with interval time varying delay has been investigated. Based in improved Lyapunov function, a delay dependent conditions for the existence of the fault estimator are given in terms of linear matrix inequalities. This result overcomes the drawbacks of delay independent results. An illustrative example has been presented to demonstrate the potential of the method.

\section{REFERENCES}

K. Gu, V. L. Kharitonov and J. Chen Stability of time delay systems. Birhauser, 2003.

B. Jiang, M. Staroswiecki, V. Cocquempot Fault accommodation for nonlinear dynamic systems. IEEE Trans. Automat. Contr. 51(9), 1578-1583 (2006).

Akhenak A. Conception dobservateurs non linaires par approche multimod'ele : application au diagnostic. $\mathrm{PhD}$ thesis, Institut National Polytechnique de Lorraine, France, 2004. 
D. Ichalal, B. Marx, J. Ragot, D. Maquin, State estimation of Takagi-Sugeno systems with unmeasurable premise variables, IET Control Theory and Applications, Vol. 4(5), pp. 897-908, 2010.

D. Ichalal, B. Marx, J. Ragot, D. Maquin, An approach for the state estimation of Takagi-Sugeno models and application to sensor fault diagnosis, Proc. of the 48th IEEE Conference on Decision and Control, CDC'09, 2009.

M. Chadli, A. Akhenak, J. Ragot, D. Maquin, State and Unknown Input Estimation for Discrete Time Multiple Model, Journal of the Franklin Institute, Vol. 346, No. 6, pp. 593-610, 2009.

M. Bouattour, M. Chadli, A. El Hajjaji, M. Chaabane, Estimation of state, actuator and sensor faults for $T$ $S$ fuzzy models, 49th IEEE Conference on Decision and Control, IEE-CDC, December 15-17, Atlanta-Georgia, USA (2010).

M. Oudghiri-Bentaie, M. Chadli, A. El Hajjaji, Robust Output $H_{\infty}$ Fuzzy Control for Active Fault Tolerant Vehicle Stability, 17th IFAC World Congress, The International Federation of Automatic Control, pp. 129-134, July 6-11, Seoul Korea (2008).

K. Zhang, B. Jiang, V. Cocquempot, Adaptive observerbased fast fault estimation. Int. J. Control Autom. Syst. 6(3), 320-326 (2008)

K. Zhang, B. Jiang, P. Shi A New Approach to ObserverBased Fault-Tolerant Controller Design for TakagiSugeno Fuzzy Systems with State Delay. Circuits Syst Signal Process, 28, 679-697 (2009)

B. Jiang, K. Zhang, P. Shi Less conservative criteria for fault accommodation of time-varying delay systems using adaptative fault diagnosis observer. I. Journal Of Adaptative Control and Signal Processing, (2009)

Zhang Y, Heng P A., Stability of fuzzy control systems with bounded uncertain delays, IEEE Transactions on Fuzzy Systems, 2002.

M. Chadli and A. El Hajjaji, A Observer-based robust fuzzy control of nonlinear systems with parametric uncertaintie. Fuzzy Sets and Systems, 2006.

M. Chilali and P. Gahinet, $H_{\infty}$ design with pole placement constraints: An LMI approach. IEEE Transactions on Automatic Control, 41(3) :358-367, 1996.

E. Kim and H. Lee, New approaches to relaxed quadratic stability condition of fuzzy control systems. IEEE Trans. Fuzzy Syst., vol. 8, no. 5, pp. 523-534, 2000.

E. Boukas and A. El Hajjaji, On Stabilizability of Stochastic Fuzzy Systems. Proceedings of the 2006 American Control Conference Minneapolis, Minnesota, USA ,p. 4362-4366, 2006.

B. Chen and X. Liu, Fuzzy guaranteed cost control for nonlinear systems with time-varying delay. IEEE Trans., vol. 13, no. 2, pp. 238-249, 2005.

K. R. Lee, J. H. Kim, E. T. Jeung, and H. B. Park, Output feedback robust $H_{\infty}$ control of uncertain fuzzy dynamic systems with time-varying delay. IEEE Trans. Fuzzy Syst., vol. 8, no. 6, pp. 657-664, 2000.

S. $\mathrm{Xu}$ and J. Lam, Robust $H_{\infty}$ control for uncertain discrete-time-delay fuzzy systems via output feedback controllers. IEEE Trans. Fuzzy Syst., vol. 13, no. 1, pp. 82-93, 2005.

C. Li, H. Wang, and X. Liao, Delay-dependent robust stability of uncertain fuzzy systems with time-varying delays. Proc. Inst. Elect. Eng. Control Theory Appl., vol. 151, no. 4, pp. 417-421, 2004.

X.-P. Guan and C.-L. Chen, Delay-dependent guaranteed cost control for T-S fuzzy systems with time delays. IEEE Trans. Fuzzy Syst., vol. 12, no. 2, pp. 236-249, 2004.

B. Chen and X. Liu, Delay-dependent robust $H_{\infty}$ control for T-S fuzzy systems with time delay. IEEE Trans. Fuzzy Syst., vol. 13, no. 2, pp. 238-249, 2005. 